BACKGROUND: Influenza virus is a worldwide health problem with significant economic consequences. To study the gene expression pattern induced by influenza virus infection, it is useful to reveal the pathogenesis of influenza virus infection; but this has not been well examined, especially in vivo study.

Aims: To assess the influence of influenza virus infection on gene expression in mice, mRNA levels in the lung and tracheal tissue $48 \mathrm{~h}$ after infection were investigated by cDNA array analysis.

Methods: Four-week-old outbred, specific pathogen free strain, ICR female mice were infected by intranasal inoculation of a virus solution under ether anesthesia. The mice were sacrificed $48 \mathrm{~h}$ after infection and the tracheas and lungs were removed. To determine gene expression, the membrane-based microtechnique with an Atlas cDNA expression array (mouse 1.2 array II) was performed in accordance with the manual provided.

Results and conclusions: We focused on the expression of $46 \mathrm{mRNAs}$ for cell surface antigens. Of these $46 \mathrm{mRNAs}$ that we examined, four (CD1d2 antigen, CD39 antigen-like 1, CD39 antigen-like 3, CD68 antigen) were up-regulated and one (CD36 antigen) was down-regulated. Although further studies are required, these data suggest that these molecules play an important role in influenza virus infection, especially the phase before specific immunity.

Key words: Influenza, cDNA array, Cell surface antigen, Cytokine, Murine

\section{Gene expression of cell surface antigens in the early phase of murine influenza pneumonia determined by a cDNA expression array technique}

\author{
Shinya Sakai ${ }^{1, C A}$, Naoki Mantani ${ }^{1,3}$, \\ Toshiaki Kogure ${ }^{1,3}$, Hiroshi Ochiai ${ }^{2}$, Yutaka Shimada ${ }^{1}$ \\ and Katsutoshi Terasawa ${ }^{1}$
}

${ }^{1}$ Department of Japanese Oriental (Kampo) Medicine and ${ }^{2}$ Department of Human Science, Faculty of Medicine, Toyama Medical and Pharmaceutical University, 2630 Sugitani, Toyama 930-0194, Japan, and ${ }^{3}$ Department of Integrated Japanese Oriental Medicine, School of Medicine, Gunma University, Gunma, Japan

CA Corresponding Author
Tel: +81764347393
Fax: +81764340366
E-mail: shinyas@ms.toyama-mpu.ac.jp

\section{Introduction}

Influenza is a major epidemic viral disease in humans. Its continuing importance lies in its ability to cause pneumonitis, which occurs in previously fit individuals and in the elderly. The infection of mice with mouse-adapted influenza virus strains by aerosol inoculation results in severe disease with alveolar involvement. The pulmonary pathology in mice is similar to that seen in viral influenza pneumonia in humans.

Although 15-20 genes have been characterized as showing increased expression after infection of the respiratory tract with influenza virus, these genes probably represent only a small fraction of all the genes that are induced under these circumstances. Little is known about the genes involved in the inflammatory response to the influenza virus. In particular, little information has been gathered about the early phase of this response, before the appearance of specific cytotoxic T cells. During this phase, the influenza virus interacts with cells on the luminal side of the airways and alveoli to induce the release of immunoactive mediators, thus initiating a cascade of events resulting in the eventual elimination of the virus. However, cell surface antigens are expressed on the cell surface and play important roles in cell-cell interactions in various situations involving the site of influenza virus infection. Examining the expression of cell surface antigens in influenza virus infection contributes important information about the network among various cells.

Recently, techniques allow characterization of the mRNA expression status of many genes. ${ }^{1,2}$ In the present study, we examined mRNA expression in murine influenza pneumonia using a cDNA array technique, especially focusing on cell surface antigens. Via this experiment, it may be understood that the molecule that until now has not seemed to take part in influenza virus infection, does actually take part in pathogenesis of the infection.

\section{Materials and methods}

\section{Preparation of the virus}

The lung-adapted strain of influenza A/PR/8/34 (PR8) virus (H1N1 subtype) was propagated in the chorioallantoic cavity of 10-day-old embryonated hen eggs for $48 \mathrm{~h}$ at $35^{\circ} \mathrm{C}$. The chorioallantoic fluid was 
Table 1. The cDNAs for cell surface antigens tested in the present study

Bone marrow stromal cell antigen 1

CD81 antigen

CD151 antigen

CD19 antigen

CD1d2 antigen

CD24a antigen

CD33 antigen

CD34 antigen

CD36 antigen

CD37 antigen

CD38 antigen

Ectonucleoside triphosphate diphosphohydrolase 1

Ectonucleoside triphosphate diphosphohydrolase 3

CD44 antigen precursor

CD48 antigen

CD6 antigen

Cd63 antigen

CD68 antigen

CD72 antigen

Immunoglobulin-associated alpha

CD8 antigen, alpha chain

CD8 antigen, beta chain

Kangai 1 (suppression of tumorigenicity 6, prostate)

CD83 antigen

CD84 antigen

CD86 antigen

\author{
CD8beta opposite strand \\ CD9 antigen \\ Coagulation factor III \\ Complement receptor-related protein \\ Duffy blood group \\ Epithelial V-like antigen \\ Tumor necrosis factor receptor superfamily, member 6 \\ Integrin- $\beta_{2}$ \\ Lymphocyte antigen 64 \\ Tumor-associated calcium signal transducer 1 \\ Lymphocyte antigen 78 \\ Interleukin-1 receptor-like 1 \\ Lymphocyte antigen 86 \\ Lymphocyte antigen 9 \\ Lymphocyte antigen 94 \\ Sialophorin \\ Tumor necrosis factor (ligand) superfamily, member 4 \\ Tumor necrosis factor receptor superfamily, member 5 \\ Tumor necrosis factor receptor superfamily, member 8 \\ Tumor rejection antigen $\mathrm{P} 1 \mathrm{~A}$ \\ CD3 antigen, delta polypeptide \\ CD3 antigen, epsilon polypeptide \\ CD3 antigen, gamma polypeptide \\ CD3 antigen, zeta polypeptide \\ CD3 antigen, zeta polypeptide
}

collected and stored in small portions at $-80^{\circ} \mathrm{C}$ after centrifugation at $1,000 \times g$ for $10 \mathrm{~min}$. The virus titer of the chorioallantoic fluid was $1.9 \times 10^{8}$ plaque forming units as determined on Mardin-Darby canine kidney cells, as described previously. ${ }^{3}$

\section{Virus infection of mice and sample preparation}

Four-week-old outbred, specific pathogen free strain, ICR female mice (body weight, $\sim 17 \mathrm{~g}$ ) were obtained from SLC Co. Ltd (Hamamatsu, Japan) and were infected by intranasal inoculation of a virus solution containing $1.0 \times 10^{4}$ plaque forming units $/ 25 \mu 1$ (five $50 \%$ lethal doses of virus) under ether anesthesia. The mice were sacrificed $48 \mathrm{~h}$ after infection and the tracheas and lungs were removed. The resected trachea and lungs were frozen by liquid nitrogen immediately after resection.

\section{cDNA expression array}

To determine gene expression, the membrane-based microtechnique with an Atlas cDNA expression array (mouse 1.2 array II; Clontech Tokyo, Japan) was performed in accordance with the manual provided. The array included 1176 mouse cDNAs and nine housekeeping control cDNAs and negative controls immobilized on a nylon membrane. The cDNAs on a membrane were divided into 23 categories including 46 cDNAs for cell surface antigens (see Table 1).

The purified RNA, which was analyzed for genomic DNA contamination by polymerase chain reaction with primers specific for $\beta$-actin, was processed with gene-specific CDS primer mix (Clontech), deoxynucleoside triphosphate, $\left[{ }^{32} \mathrm{P}\right] \mathrm{dATP}$, and reverse transcriptase for preparation of cDNA. The ${ }^{32} \mathrm{P}$-labeled cDNA was purified through a Chroma Spin-200 column (Clontech). The labeled cDNA in a solution of ExpressHyb (Clontech) with heat-denatured, shearedsalmon-test DNA was then hybridized overnight to the Atlas array membrane at $68^{\circ} \mathrm{C}$. The membrane was washed in $2 \mathrm{x}$ standard saline citrate (SSC) ( $1 \mathrm{x}$ SSC is $0.15 \mathrm{M} \mathrm{NaCl}+0.015 \mathrm{M}$ sodium citrate) with $1 \%$ sodium dodecyl sulfate, in $0.1 \times$ SSC with $0.5 \%$ sodium dodecyl sulfate, and in $2 \times$ SSC, sequentially, and was then exposed to PhosphorImager (Storm 860; Molecular Dynamics, Sunnyvale, CA, USA). Results of the gene expression were analyzed by computer using Atlas imaging software (Clontech).

\section{Statistic analysis}

The comparison of gene expression findings between non-infected control mice and infected mice was analyzed by Student's $t$ test.

\section{Results and discussion}

Table 1 presents the cDNAs for cell surface antigens that were tested in the present study. Of 46 mRNAs examined, four were up-regulated and one mRNA was down-regulated $48 \mathrm{~h}$ post infection (Fig. 1). Gene expression levels (spot intensity) of CD1d2 antigen, CD39 antigen-like 1, CD39 antigen-like 3 and CD68 antigen before infection were $3.7 \pm 1.86,2.0 \pm 0,3.0 \pm 0$ and $4.0 \pm 1.63$, respectively. At $48 \mathrm{~h}$ after infection, gene 


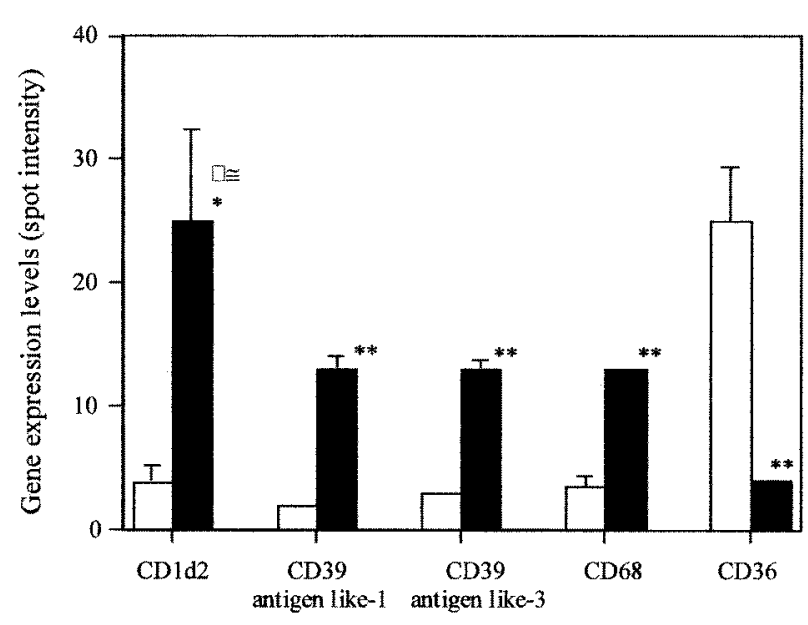

FIG. 1. The mRNA expression levels for cell surface antigen in the lung and trachea tissue of influenza-infected mice. Results are presented as the mean \pm SE from three independent experiments. Open column, before infection; filled column, 48-h after infection of influenza virus. ${ }^{*} p<0.05 * * p$ $<0.01$ compared with non-infected controls, analyzed by Student's $t$-test.

expression levels of CD1d 2 antigen, CD39 antigen-like 1, CD39 antigen-like 3 and CD68 antigen were $25.0 \pm 7.76,12.0 \pm 1.63,13.0 \pm 1.23$ and $13.0 \pm 0.41$, respectively. Gene expression levels of CD36 antigen before infection and $48 \mathrm{~h}$ after infection were $24.03 \pm 6.54$ and $4.0 \pm 0.82$, respectively. Results are presented as the mean (SE from three independent experiments (each experiment was carried out once).

In the present study, we examined the expression of 46 cell surface antigen mRNAs. The expression of four mRNAs (CD1d2 antigen, CD39 antigen-like 1, CD39 antigen-like 3, and CD68 antigen) were up-regulated. CD36 antigen was down-regulated (Fig. 1).

CD1d demonstrated an important role in natural killer (NK) T-cell development. ${ }^{4-7}$ NK T cells release large amounts of IL- 4 within hours of activation, and it has been proposed that this population of $\mathrm{T}$ cells may be the earliest source of IL- 4 that could shift an immune response toward a $\mathrm{T}$ helper cell type 2 phenotype., ${ }^{5,8}$ However, the mechanisms of NK T-cell stimulation and the function of CD1d is not fully understood. This finding provides a further clue to understanding these mechanisms.

CD39 regulates vascular inflammation and thrombosis by hydrolyzing adenosine triphosphate and adenosine diphosphate. Recently, CD39 has been identified as responsible for Langerhans cell-associated ectoNTPDase activities, and contradictory outcomes of CD39 deficiency have been demonstrated in skin inflammation and immune responsiveness. ${ }^{9}$

CD68 is expressed on macrophages, monocytes, neutrophils, basophils and lymphocytes, and is especially useful as a marker for monocytes, but its function is not well understood. ${ }^{10-12}$

CD36 is known as one of a Class B scavenger receptor family, a candidate for the receptor of phosphatidylserine, which plays an important role in the recognition and phagocytosis of senescent and apoptotic cells. ${ }^{13-15}$ In the present study, expression of CD36 mRNA was down-regulated.

In the present study, we have demonstrated that the expression of some cell surface antigen mRNAs were up-regulated or down-regulated $48 \mathrm{~h}$ after influenza virus infection in the lung and trachea of mice. The relation to the influenza virus infection of these molecules has not been pointed out until now. Why the mRNA expression of these molecules is neither up-regulated nor down-regulated is understood only by this experiment. However, new information to elucidate the pathogenesis of influenza pneumonia might be discovered by researching these molecules and the relations to infection in the future. A cDNA array technique is a useful tool to obtain information such as the biological response to viral infections in a pilot study.

\section{References}

1. Coombes BK, Mahony JB. cDNA array analysis of altered gene expression in human endothelial cells in response to Chlamydia pneumoniae infection. Infect Immun 2001; 69: 1420-1427.

2. Eckmann L, Smith JR, Housley MP, Dwinell MB, Kagnoff MF Analysis by high density cDNA arrays of altered gene expression in human intestinal epithelial cells in response to infection with the invasive enteric bacteria Salmonella. J Biol Chem 2000; 275: 14084-14094.

3. Ochiai H, Ikesue A, Kurokawa M, Nakajima K, Nakagawa H. Enhanced production of rat interleukin-8 by in vitro and in vivo infections with influenza A NWS virus. J Virol 1993; 67: 6811-6814.

4. Bendelac A, Rivera MN, Park SH, Roark JH. Mouse CD1-specific NK1 T cells: development, specificity, and function. Ann Rev Immunol 1997; 15: 535-562.

5. Chen YH, Chiu NM, Mandal M, Wang N, Wang CR. Impaired NK1+T cell development and early IL-4 production in CD1-deficient mice. Immunity 1997; 6: 459-467.

6. Mendiratta SK, Martin WD, Hong S, Boesteanu A, Joyce S, Van Kaer L. CD1d1 mutant mice are deficient in natural $T$ cells that promptly produce IL-4. Immunity 1997; 6: 469-477.

7. Smiley ST, Kaplan MH, Grusby MJ. Immunoglobulin E production in the absence of interleukin-4-secreting CD1-dependent cells. Science 1997; 275: $977-979$.

8. Denkers EY, Scharton-Kersten T, Barbieri S, Caspar P, Sher A. A role for CD4+ NK1.1+ T lymphocytes as major histocompatibility complex class II independent helper cells in the generation of CD8+ effector function against intracellular infection. J Exp Med 1996; 184: 131-139.

9. Mizumoto N, Kumamoto T, Robson SC, Sevigny J, Matsuse H, Enjyoji K, Takashima A. CD39 is the dominant Langerhans cell-associated ectoNTPDase: modulatory roles in inflammation and immune responsiveness. Nat Med 2002; 8: 358-365.

10. Ruco LP, Pulford KA, Mason DY, et al. Expression of macrophageassociated antigens in tissues involved by Langerhans' cell histiocytosis (histiocytosis X). Am J Clin Pathol 1989; 92: 273-279.

11. Brandtzaeg P, Dale I, Gabrielsen TO. The leucocyte protein L1 (calprotectin): usefulness as an immunohistochemical marker antigen and putative biological function. Histopathology 1992; 21: 191-196.

12. McGuinness PH, Painter D, Davies S, McCaughan GW. Increases in intrahepatic CD68 positive cells, MAC387 positive cells, and proinflammatory cytokines (particularly interleukin 18) in chronic hepatitis C infection. Gut 2000; 46: 260-269.

13. Albert ML, Pearce SF, Francisco LM, Sauter B, Roy P, Silverstein RL, Bhardwaj N. Immature dendritic cells phagocytose apoptotic cells via alphavbeta5 and $\mathrm{CD} 36$, and cross-present antigens to cytotoxic $\mathrm{T}$ lymphocytes. J Exp Med 1998; 188: 1359-1368.

14. Shiratsuchi A, Kawasaki Y, Ikemoto M, Arai H, Nakanishi Y. Role of class B scavenger receptor type $\mathrm{I}$ in phagocytosis of apoptotic rat spermatogenic cells by Sertoli cells. J Biol Chem 1999; 274: 5901-5908.

15. Tait JF, Smith C. Phosphatidylserine receptors: role of CD36 in binding of anionic phospholipid vesicles to monocytic cells. J Biol Chem 1999; 274: 3048-3054. 


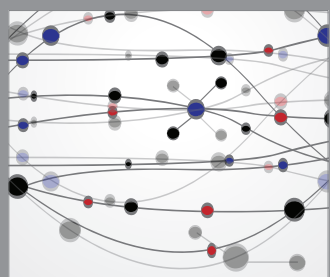

The Scientific World Journal
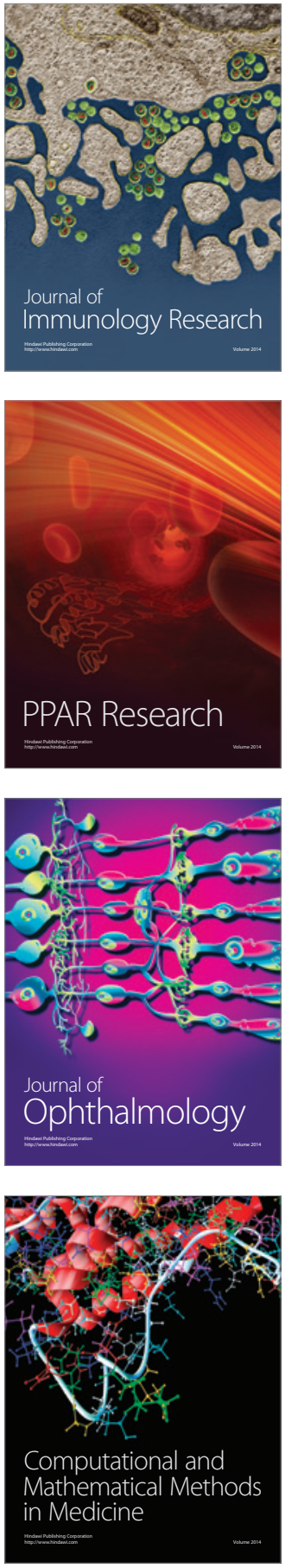

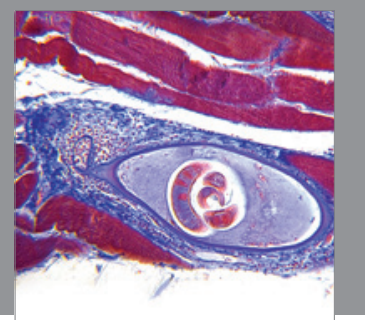

Gastroenterology

Research and Practice
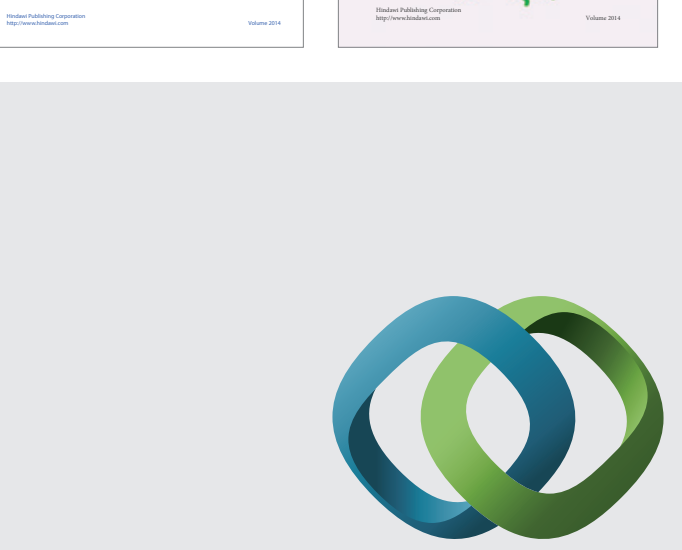

\section{Hindawi}

Submit your manuscripts at

http://www.hindawi.com
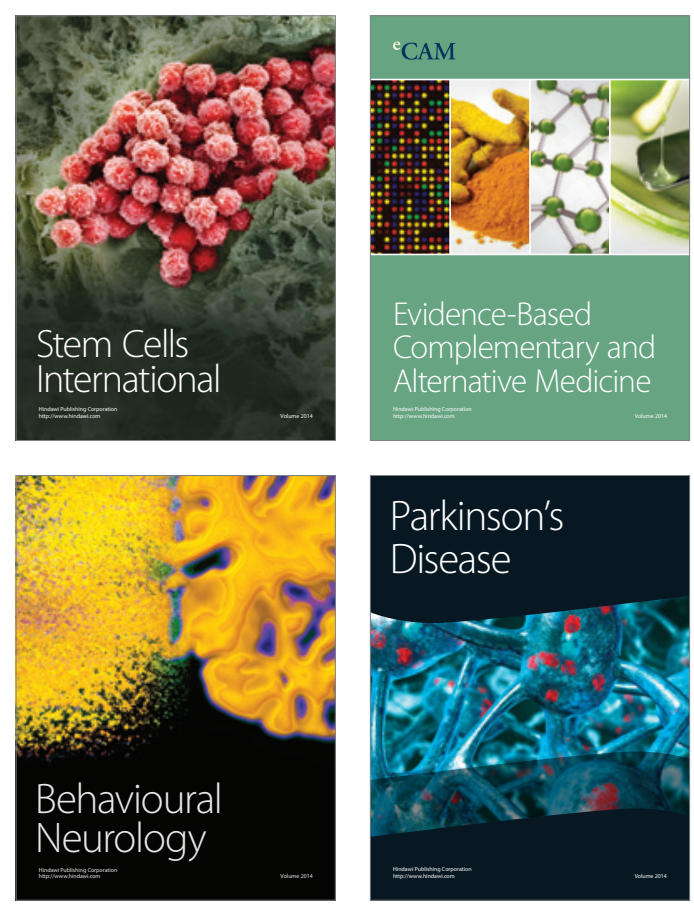

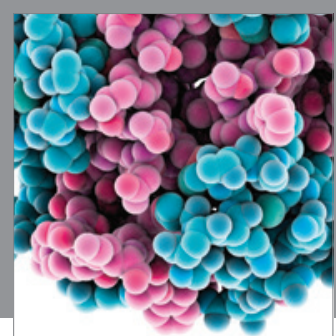

Journal of
Diabetes Research

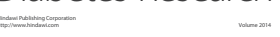

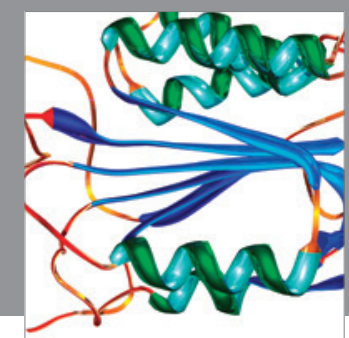

Disease Markers
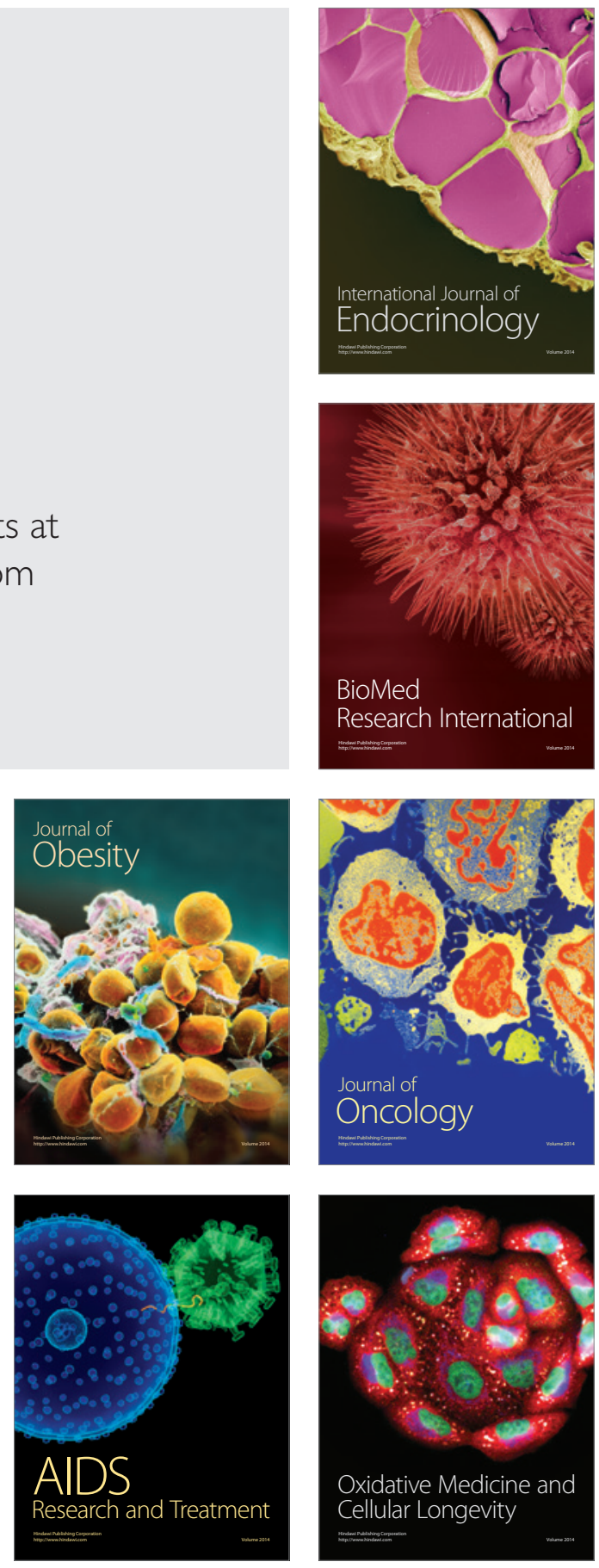